\title{
Un regard sur les recherches roumaines en histoire de la traduction
}

\author{
Georgiana LUNGU-BADEA \\ Université de l'Ouest, Timisoara \\ Roumanie
}

\begin{abstract}
Résumé : Dans cet article, nous nous proposons de présenter un panorama de l'histoire et de l'historiographie de la traduction en roumain, dans le concert des multiples approches dont l'histoire de la langue, l'histoire de la littérature et l'histoire des pratiques artistiques et culturelles peuvent faire l'objet. Pour réaliser ce survol, nous avons établi comme repères : la traduction comme acte, les acteurs de la traduction (partiellement recensés dans des répertoires et/ou indirectement ordonnés dans des dictionnaires et répertoires des œuvres traduites) et la traduction comme résultat. Notre intention est de mettre en avant l'utilité des recherches en histoire de la traduction roumaine, qui servent principalement à révéler le rôle que la traduction a joué dans l'autodétermination linguistique et administrative et, par cela, dans la construction du passé national.
\end{abstract}

Mots clés : traduction(s), traducteurs, histoire de la traduction en roumain/de la langue roumaine, historiographie de la traduction.

\begin{abstract}
This article sets out to offer a panoramic view of the history and historiography of translation into Romanian from a variety of approaches, among them the history of language, of literature, and of cultural and artistic practices. The following aspects will be focused on in carrying out this survey: translation as an act, the participants in the translation process (partially recorded in indexes and/or indirectly listed in dictionaries and indexes of translated works), and translation as a result. Our intention is to emphasize how valuable research in the field of Romanian translation is, in particular in order to reveal the role translation has played in the process of linguistic and administrative self-determination, and, through this, in the construction of the national past.
\end{abstract}

Keywords : translation(s), translators, history of translation into/from Romanian, historiography of translation.

\section{Introduction}

En Roumanie, on peut parler des débuts d'une discipline scientifique, l'historiographie de la traduction, fondée sur les recherches en histoire de la traduction et le modèle de continuité temporelle (le recensement des traductions, la description chronologique des traductions, des traducteurs) où la périodisation est empruntée à l'histoire de la langue et/ou de la littérature roumaine. À 
cela vient s'ajouter une réflexion sur le métalangage de la traduction (stratégies, procédés, méthodes, concepts, etc.) et sur les visées de la recherche en histoire et, ensuite, en historiographie de la traduction.

La réflexion que nous proposons dans les pages qui suivent, très générale, n'est qu'une esquisse pour une historiographie de la traduction en roumain. Les limites qu'elle comporte dérivent aussi bien du fait que l'histoire de la traduction en roumain n'a pas encore atteint son point culminant, que de la difficulté d'inventorier les sources imprimées et électroniques. Cependant, dans les recherches roumaines en histoire de la traduction examinées, nous avons pu identifier des points de référence selon lesquels on s'accorde tacitement à envisager l'histoire de la traduction en roumain. Ces repères, bien ancrés dans l'histoire nationale et la culture roumaine, dans l'évolution de la langue et de la littérature roumaines, sont complétés par la présentation des contacts livresques (lecture des textes originaux, des textes traduits) et des contacts non livresques, directs ${ }^{1}$ et indirects ${ }^{2}$.

Conséquemment, on observe le décalage entre deux histoires : l'« histoire interne », vécue et écrite par " ceux qui la produisent », et l'« histoire externe », " élaborée rétrospectivement par des chercheurs modernes » (D’hulst 2014, 17). Bien que ce décalage soit clair, les chercheurs roumains ne répondent que rarement et, le plus souvent, implicitement à des questions comme : "À quoi (qui) sert l'histoire de la traduction ? ", "Faut-il envisager une histoire générale de la traduction - « des grands panoramas internationaux » (Delisle 1998, 21-43) - dans laquelle l'histoire nationale trouve une/sa place ? », ou "Faut-il d'abord élaborer une histoire nationale des traductions /de la traduction/des traducteurs? ».

En considérant largement le champ de la traduction, nous tentons de traiter impartialement les axes de recherches historiques, historiographiques et métahistoriographiques (cf. D’hulst 2014, 22). Nous posons, dans la présente esquisse historiographique, des jalons la traduction, les traductions et les traducteurs - susceptibles de permettre : l'identification des connexions qui s'instaurent entre les théories inductives de la traduction (des XVIII e et XIX ${ }^{\mathrm{e}}$ siècles), l'emprunt des modèles théoriques d'Occident et les théories

\footnotetext{
${ }^{1}$ Il s'agit des contacts internes non seulement en Transylvanie et dans le Banat, mais aussi en Valachie et en Moldavie où s'installent des Français réfugiés à l'Est après 1789 , des précepteurs, marchands, etc. Même réduits, les contacts avec les Anglais qui voyagent dans les provinces roumaines, au XIXe siècle, sont recensés pour avoir contribué à faire connaître les Roumains aux Anglais (v. Iorga 1917, M. Beza, English Travellers in Romania, 1920, cités par Grimm 2012, 27) ; et des contacts externes, établis par des jeunes Roumains partis étudier en France, Italie, dans les villes de l'empire des Habsbourg.

2 Avec la culture, la littérature et la langue françaises, entre autres, par l'intermédiaire des phanariotes grecs, des officiers et soldats russes, etc.
} 
linguistiques déductives ; l'examen du rapport qui existe entre l'histoire et l'historiographie, la théorie et la pratique de la traduction.

\section{L'historiographie de la traduction en roumain}

En Roumanie, le travail d'historien et/ou d'historiographe est entrepris par des chercheurs qui proviennent d'horizons traductifs différents : des enseignants de la traduction/de la littérature (Grimm [1916-1943]2012, Jacquier 1991), des critiques de la traduction, des traductologues. Certains chercheurs s'intéressent au rôle que la traduction a joué dans l'histoire des Roumains et à sa contribution à la compréhension de l'histoire, rôle qu'elle ne cesse pas d'accomplir; d'aucuns visent l'analyse historique des méthodes et formes de traduction et, enfin, d'autres s'attachent à dépeindre les traducteurs ${ }^{3}$ et à étudier les conditions dans lesquelles ceux-ci ont travaillé. La périodisation historique varie d'une recherche à l'autre, selon que les chercheurs prennent en compte les histoires de la langue et/ou de la littérature roumaine ${ }^{4}$, les langues traduites ${ }^{5}$, les courants ${ }^{6}$ et les genres littéraires traduits ${ }^{7}$, etc. Tout récemment, on a pu constater une tendance non seulement à rehausser et à expliquer des méthodes de traduction anciennes, mais aussi à développer des méthodes chronologique, thématique, générique, etc., et des instruments de recherche spécifiques ${ }^{8}$ à l'historiographie de la traduction.

La manière dont la traduction a influé sur la formation de la langue roumaine littéraire, sur la création de la littérature roumaine a déjà fait l'objet d'étude des ouvrages entièrement ou partiellement consacrés au poids qu'elle a eu dans la compréhension et la détermination de différents phénomènes historiques. Ce sont les

3 Voir aussi Dicționarul literaturii române de la origini pînă la 1900 [Dictionnaire de la littérature roumaine, des origines à 1900], Academia R.S.R., Universitatea "Al. I. Cuza" din Iași. Institutul de Lingvistică, Istorie Literară și Folclor, 1979.

4 Nous ne notons ici que quelques-uns des noms d'historiens susceptibles d'influencer les recherches en histoire de la traduction: Al. Philippide 1888 ; E. Lovinescu 1926-1929; G. Călinescu 1941; G. Ivaşcu 1969; S. Cioculescu 1973

5 Les répertoires des traducteurs et des traductions roumaines des langues française, italienne, espagnole (RTR I et RTR II, 2006).

6 Mircea Anghelescu, Preromantismul românesc [Le Préromantisme roumain]. București: Minerva, 1971

7 Dicţionarul romanului tradus în limba română [Dictionnaire du roman traduit en langue roumaine] 2005. Mihai Eminescu, Teatrul original și tradus. Traducerile de proză literară. Dicționarul de rime [Théâtre original et traduit. Traductions de prose littéraire. Dictionnaire de rimes]. Volum îngrijit de Perpessicius. București: Fundația pentru Literatură și Artă "Regele Carol II", 1988.

8 Il s'agit des répertoires, dictionnaires, anthologies, biographies, bibliographies, iconographies, etc. 
historiens de la langue roumaine qui se sont penchés les premiers sur l'étude de la traduction, et l'historiographie du roumain est déjà constituée. Leurs approches ont facilité la compréhension de la traduction en tant que phénomène et des rapports qu'elle a entretenus avec d'autres activités artistiques et éducatives, politiques et administratives; elles ont également ouvert la voie aux approches strictement historiques de la traduction dont nous tentons de donner ici une première esquisse historiographique.

\subsection{Remarques sur l'histoire de la traduction en roumain et les béhaviourèmes des traducteurs}

Une série de recherches roumaines en histoire de la traduction enchaînent chronologiquement les évènements de l'histoire générale et interprètent leur signification dans la pratique de la traduction. Ainsi la réception et l'influence des idées européennes ont-t-elles été déterminantes dans l'histoire des Roumains. De grands moments et actes, étudiés en rapport avec la traduction, ont aidé à comprendre l'évolution de la langue, de la culture et de la société roumaines, à mesurer leur impact sur le progrès et à construire l'histoire de ce peuple. Il s'agit donc des recherches qui ont adopté la chronologie des événements et estimé l'impact qu'ont eu sur la traduction les réformes scolaires de 1770 et 1790 ; la création des écoles où l'enseignement était dispensé en roumain ; la déclaration de l'allemand en tant que langue officielle en Transylvanie, ce qui a mené à l'institutionnalisation du bilinguisme roumain-allemand (1795); la participation des boyards roumains bilingues natifs (gréco-roumains) à la renaissance de l'hellénisme ; de la révolution de Tudor Vladimirescu, 1821 ; le Traité de paix d'Andrinople (1829) ; le passage au bilinguisme franco-roumain, après le Règlement organique (1831 en Moldavie, 1832 en Valachie) ; la révolution roumaine de 1848 ; l'union de la Moldavie et de la Valachie (1859). Dans ces circonstances, directement et indirectement, des littératures et savoirs d'ailleurs ont pénétré dans la langue et la culture roumaines (Iorga 1907-1909, Piru 1977, Gheţie 1978, Ivănescu 1980, Munteanu et Târa [1978] 1983).

L'étude historique du processus traductif relève que les traductions-résultats ressortent d'épistèmês différents et que, pour réaliser le panorama de l'histoire et de l'historiographie de la traduction roumaine, il est nécessaire de partir du contexte politique et économique, d'une part, et du contexte scientifique, littéraire, artistique, d'autre part. Les relations culturelles, interculturelles et transdisciplinaires ont déterminé le paradigme traductif et les changements que celui-ci a subi sous l'influence des tendances externes et internes variées (Philippide 1888, Lovinescu 1926-1929, Călinescu 1941, Ivaşcu 1968, Cioculescu 1973). Ainsi les historiographes ont-ils été amenés à s'interroger sur les visées présomptives de l'historien et sur sa réflexion sur le métalangage historique, et, subséquemment, à 
établir le lien entre la conception traductive du passé et celle du présent (Grimm [1916-1943] 2012, Ursu 1962, Munteanu 1995, Loría-Rivel 2004, Lungu-Badea 2007, 2008, 2013, Lenz 2011, Gafton 2012). Enfin, ils ont montré que les réflexions descriptives, tout comme les théories linguistiques (latinisme, purisme, italianisme) et l'intention de normaliser la langue roumaine et la traduction en roumain représentent le bien-fondé de la recherche en histoire de la traduction en Roumanie. C'est grâce à l'acte de déshistorisation qu'il a été possible, d'abord, de tracer les axes de la pensée prototraductologique roumaine, ensuite, d'identifier et d'étudier les racines et raisons du développement actuel de la traductologie en Roumanie, et, enfin, de proposer une esquisse de l'historiographie de la traduction et des traductions en roumain (Lungu-Badea, 2013).

Dans un autre volet de l'historiographie de la traduction, se situent l'analyse de préfaces, de discours des traducteurs (Gafton 2012, Giurginca 2012) et de textes traduits (Iorga 1936, Anghelescu 1972, 1974, Barbu 1987), la traduction comparée, les monographies centrées sur des traducteurs et œuvres traduites (Gheţie et Mares 1996, Isar 2003, Camariano 1946, Ursu 1994), sur des écrivains traduits en roumain ${ }^{9}$, ou sur des auteurs roumains traduits dans d'autres langues. ${ }^{10}$

Ces recherches et bien d'autres, que les contraintes spatiales de cet article nous empêchent de citer ici, ont pu contextualiser les problèmes et difficultés auxquels se sont heurtés les traducteurs; montrer les corrélations qui existent entre les discours, les objectifs et les instruments des traducteurs roumains d'antan et d'aujourd'hui. On a pu constater des dissemblances (dues, de toute évidence, au décalage temporel et au décalage intra- et interlinguistique) et des similitudes entre les béhaviourèmes traductifs et prototraductologiques des traducteurs roumains et des traducteurs occidentaux, allant des méthodes aux stratégies et procédés (Lungu-Badea 2013).

À la complexité de l'historiographie roumaine de la traduction participent les monographies, plus rares, et les portraits de traducteurs, les articles des dictionnaires sur les traducteurs. Des chercheurs roumains ont examiné la manière des traducteurs de contribuer à introduire des écrivains étrangers, à offrir des modèles aux écrivains autochtones, à vulgariser les savoirs, à former le goût du public

9 Dans Lermontov, Editura Tineretului, Bucureşti, Colecția „Oameni de seamă”, 1963, Tamara Gane analyse les traductions roumaines de Lermontov. L'ouvrage contient également un répertoire des œuvres de Lermontov traduites en roumain. Dans la foulée de Gane, Elena Cervinschi présente dans son ouvrage, Lermontov în România, București, Editura Minerva, 1987, la qualité des traductions, les causes des erreurs et leurs effets sur le lecteur. Le deuxième tome des œuvres complètes d'Alexandru Macedonski réunit les traductions faites du français et offre de la sorte des renseignements explicites sur l'intérêt traductif de l'écrivain.

${ }^{10}$ Voir Elena Loghinovski, Eminescu în limba lui Puşkin, Iaşi, Junimea, 1989. 
roumain cible et à l'éduquer. Ils ont montré que, quel que soit le statut des auteurs traduits (d'anonyme, de commun ou de notoriété et, par conséquent, récompensés et/ou critiqués), les traducteurs se nourrissent aussi de la gloire des auteurs traduits. Ainsi, on peut constater que dans l'espace de réception du roumain-cible, la renommée du traducteur est soit immanente (Beldiman, Heliade Radulescu, Spartali, Marcovici), soit résultante de la renommée dont jouit l'auteur traduit. ${ }^{11}$

\subsection{Vues sur les recherches en histoire des traductions}

Lorsqu'on traite de l'histoire de la traduction (acte, phénomène), on envisage inévitablement l'histoire des traductions et des traducteurs, de la société où l'on a accompli l'acte de traduire. Néanmoins, il ne s'agit pas que de décrire les textes traduits et les manières de les rendre en roumain, mais plutôt de surprendre les faits, les actes, les situations dans leur évolution, afin de mieux les appréhender et apprécier. Les chercheurs insistent aussi bien sur la nouveauté - pour l'époque et pour le destinataire - des traductions que sur leur filiation (Philippide 1888, Călinescu 1941, Munteanu et Tara, 1983, Munteanu, 1995). D’ici même la nécessité de faire l'histoire de la traduction, ensuite de l'enseigner pour la faire connaître dans la complémentarité des liens qu'elle entretient avec l'histoire de la langue roumaine et l'histoire des Roumains et de leur relation avec d'autres peuples (Iorga 1917, Camariano 2008). Grâce à la traduction, on a suivi l'accomplissement des aspirations nationales, administratives politiques, littéraires et esthétiques ; grâce aux traductions, on a formé le goût des lecteurs et on les a éduqués; par l'histoire et l'historiographie de la traduction, on apprend à mieux préparer l'avenir de la langue roumaine, et à préserver son identité au moyen de la traduction.

La comparaison de l'histoire des traducteurs roumains et de l'histoire des traductions roumaines, montre que la seconde est mieux articulée. Permettant de comprendre la genèse de la littérature roumaine, les traductions littéraires ont été inventoriées dans divers types d'ouvrages : des dictionnaires (de la littérature romaine, 1979 ; du roman traduit en roumain, 2005); des répertoires des traductions et des traducteurs (RTR I et RTR II 2006), des bibliographies des œuvres littéraires traduites en roumain (Fecioru 1937, Reischel 1967,

11 Parmi les auteurs les plus traduits en roumain, on trouve : A. France, Al. Daudet, Dumas père, Fénelon, V. Hugo, G. Leopardi, P. Loti, G. Maupassant, Molière, C. Mendès, G. Sand, E. Sue, Shakespeare et d'autres. Byron, Dante, Flaubert, Thomas Gray, Mérimée, Musset, Pétrarque, Al. Pope, Racine, Voltaire, Young, etc. ont l'objet des traductions plutôt parcimonieuses ; Diderot, Métastase, des traductions espacées (cf. RTR II, 2006). On a généreusement traduit des écrivains obscurs, les traducteurs partageant leur destin. 
Geambaşu (coord.) 2011). Cette portée de la traduction est à l'origine de l'attention particulière que les chercheurs ont prêtée aux rapports établis entre la littérature roumaine et d'autres littératures, rapports consignés dans les sept tomes de la bibliographie des relations instaurées entre la littérature roumaine et les littératures étrangères dans des périodiques (1980-1985 et 1997-2002). En dépit du manque de concordance existant entre l'apparition et l'évolution de la littérature roumaine et des littératures occidentales, sa genèse ne diffère pas de la genèse d'autres littératures en langues nationales (la traduction en roumain comme genre littéraire, pratique empruntée à la France où elle a connu sa grandeur et sa décadence, allait parcourir le même chemin).

Les historiens ${ }^{12}$ de la langue roumaine ont indirectement esquissé l'histoire de la traduction; on pourrait lire, de manière détournée, l'histoire de la langue roumaine comme histoire de la traduction en roumain. Au XIX ${ }^{\mathrm{e}}$ siècle, ils étaient nombreux les lettrés roumains, historiens, grammairiens et traducteurs qui envisageaient la traduction comme un instrument d'enrichissement de la langue roumaine et, ensuite, de sa normalisation (voir, par exemple, Heliade Rădulescu [1828]1980, XXVII-XXVIII). Ils y entrevoyaient, sous l'influence du nationalisme européen, un moyen de contribuer à l'affirmation de la nation roumaine. À l'instar des historiens de la langue roumaine, les chercheurs qui s'intéressent à l'étude historique de la traduction, de la terminologie ${ }^{13}$, soulignent tantôt implicitement, tantôt explicitement l'appétence du roumain, une langue encore jeune au XIX ${ }^{\mathrm{e}}$ siècle, pour la création authentique, son ardeur d'emboîter le pas des grandes cultures (Cosco 1934 ; Iorga 1936 ; Camariano 1946 ; Nicolescu 1958 ; Anghelescu 1974; Barbu 1987; Ursu 1994; Munteanu 1995; Lenz 2011) et d'affirmer, enfin, son autonomie de création et son originalité (Lungu Badea 2013).

Les histoires de la langue et de la littérature roumaine, les monographies consacrées à des lettrés des $\mathrm{XVI}^{\mathrm{e}}-\mathrm{XIX}^{\mathrm{e}}$ siècles, les analyses philologiques des textes traduits (Moraru 1996, Anghelescu 1974, Isar 2003, Ursu 1999) permettent d'établir la filiation des idées, d'attester l'affiliation des traducteurs, de mettre en évidence la complémentarité des approches qu'exploite l'historien de la traduction

\footnotetext{
12 Nous n'en citons que quelques noms : Iorga 1907-1909; Puscariu [1920] 1989 ; Piru 1977 ; Gheţie 1978 ; Munteanu et Tara [1978] 1983 ; Ivănescu 1980. 13L'intérêt pour l'histoire de la terminologie roumaine est grandissant. N. A. Ursu (1962); Coman Lupu (2000); Gheorghe Chivu, Limba română de la primele texte până la sfârşitul secolului al XVIII-lea. Variantele stilistice (2000) ; Mihaelei Marcu, Evoluția terminologiei gramaticale românești în perioada 1757 - 1877 (2005) ; Laura Elena Pascale, Terminologia lingvistică în primele gramatici româneşti (2011); Manuela Saramandu, Terminologia juridic-administrativă românească în perioada 1780 - 1850 (1986) ; Victor Vascenco, Probleme de terminologie lingvistică (1975), ce ne sont que quelques titres d'une liste plus longue.
} 
qui tente de restaurer le rapport entre les aspirations et les intérêts politiques, nationaux et administratifs, d'une part, et les idéaux linguistiques et littéraires. Parfois conflictuels, les idéaux linguistiques, certainement complémentaires, ont servi «l'ambition d'organiser le gouvernement afin d'obtenir l'autodétermination politique et administrative, et le désir de forger un seul et unique instrument de communication pour parvenir à l'autodétermination linguistique » (Lungu-Badea 2011, 42-43). Ils ont également constitué les axes principaux des actions qu'ont menées les hommes politiques, les historiens et les écrivains du XIX siècle, tels que Gh. Asachi, I. Heliade Rădulescu, Alecu Russo, George Bariţiu, Titu Maiorescu.

L'étude historique des traductions a permis d'établir un parallèle, tellement souhaité par les lettrés roumains du XIX ${ }^{\mathrm{e}}$ siècle, entre leurs aspirations et celles des gens de lettres et des traducteurs français du XVIII ${ }^{e}$ siècle. Sans approfondir des tendances en usage en France, s'intéressant moins à l'universalité de la pensée (selon l'acception véhiculée à l'époque: extralinguistique et, donc indépendante de la langue dans laquelle celle-ci a été formulée) et à la restitution de la pensée-source, des idées de l'auteur, à l'aide des moyens dont le roumain-cible dispose, les traducteurs ont intuitivement compris et mis en œuvre le principe de traduire comme si l'auteur avait écrit en roumain. Le discours - des bribes de théorie - portant sur les techniques de traduire, marie justification des choix traductifs et invocation à la clémence des lecteurs face aux éventuelles erreurs de traduction. L'examen des préfaces qui accompagnent les traductions (Gafton 2012 ; Giurginca 2012 ; Un capitol de traductologie românească [Un chapitre de traductologie roumaine], 2008, Lungu-Badea 2013) prouvent l'utilité de la recherche historique et de la démarche d'investigation descriptive et comparée qui contribuent à éclairer un aspect insuffisamment étudié, mais significatif pour comprendre la dynamique cohérente de la littérature et de la langue roumaines. Outre les enjeux politiques et linguistiques de la traduction, les chercheurs considèrent aussi la préoccupation des traducteurs pour l'esthétique. Etant donné que la traduction a été orientée surtout vers le lecteur-cible, l'esthétique est centrée sur la réception en roumain-cible. Les attentes lectorielles, réelles ou attribuées, influent sur les objectifs et instruments de traduction (Lungu Badea 2008, 259-270). On parvient à cette conclusion après avoir examiné les traductions et les discours préfaciels qui les accompagnent. Le destin des préfaces qui accompagnent les traductions en roumain est pareil au destin des préfaces écrites en Occident, mais peu connu en tant que tel. Ces préfaces, qui ressemblent aux préfaces d'Oresme (à la traduction des traités d'Aristote), de John Dryden (à la traduction aux Epîtres d'Ovide, 1680), pourraient acquérir le statut de "petit traité de traduction». Leurs échos, fonctions et effets le confirment. Des prises de position absolues et contradictoires, orientées soit vers la langue roumaine, soit vers les langues traduites, sillonnent les 
écrits de Heliade Rădulescu, Bariţiu, Kogălniceanu, Maiorescu, etc. La politique linguistique et l'intérêt commercial des éditeurs ont agi sur l'entreprise de normalisation du roumain et de fixation d'une norme supradialectale à partir des différents dialectes roumains. L'étude des discours sur la traduction a explicitement relevé l'existence de deux aspects : d'abord, une critique dure des choix superficiels et aléatoires des écrivains mineurs ou obscurs traduits en roumain, et, ensuite, un soutien pour la création authentique (théâtre, etc.), non porteuse de marques d'étrangéité. Les arguments invoqués reviennent en somme à dire que la langue roumaine était porteuse d'une virtualité dont la concrétude aurait pu être rendue visible par un double mouvement consistant à gommer la rusticité du vocabulaire des œuvres authentiques, d'une part, et à importer de meilleurs modèles (français, italiens, allemands), d'autre part. Ce sont les principales raisons qui ont déterminé Kogălniceanu à publier, dans Dacia literară, un appel à limiter le nombre de traductions (Kogălniceanu [1840-1844] 1956). Il a critiqué le mauvais choix des textes à traduire (Cornea 1966, 51-54), et exigé qu'on ne procédât pas à la traduction d'une œuvre avant qu'on n'eût décidé de l'utilité de sa traduction pour la culture roumaine. Il a violemment condamné les imitations qui «tuai[en]t l'esprit national » (Kogălniceanu [1840] 1956, 135), l'idée de parasitage du génie national allant être reprise dans le manifeste qu'il a publié dans la revue Propăşirea ([Le Progrès] 1844). Il a néanmoins encouragé la traduction d'œuvres majeures qu'il considérait indispensables pour le progrès social des Roumains, telles que l'Esprit des lois de Montesquieu, les œuvres de Démosthène, Xénophon, Thucydide, ou la traduction de textes législatifs. L'examen des discours sur la traduction relève l'existence et la pratique des deux méthodes traditionnelles de traduction dont témoigne l'existence de la traduction universelle : une méthode/manière centrée sur la lettre et orientée vers la langue-source, l'autre sur l'esprit, le lecteur-cible et/ou la langue-cible.

L'histoire de l'histoire de la traduction, l'historiographie, permet, premièrement, de fixer des points cruciaux dans l'évolution et la pratique traductive et des points de comparaison entre les étapes de l'histoire de la traduction en Occident et l'histoire de la traduction en roumain, et secondairement, de valoriser le passé traductif, la réflexion traductive sur laquelle se fonde la recherche en traductologie actuelle.

\section{Conclusion}

Sans prétendre nous situer dans la temporalité du traducteur/de la traduction, ni dans la temporalité des contemporains de la recherche historique, nous avons essayé de les gérer et nous nourrissons l'espoir que les jalons posés ici serviront à développer une historiographie de la traduction en roumain. Afin de faciliter la compréhension des expériences du passé, nous avons suivi à saisir et à rendre l'historicité 
des faits traductifs, des réflexions sur les discours des traducteurs. Ainsi avons-nous pris appui sur celles-ci et créé le contexte favorable pour que le lecteur contemporain rencontrât l'altérité des traducteurs et des lecteurs du passé. Si le traducteur est un passeur de mots, l'historien est un passeur de faits, d'actes ; les deux facilitent la tâche des lecteurs qui tentent d'établir un lien entre le passé et le présent, entre l'ailleurs et l'ici. L'histoire de la traduction peut éclairer ainsi le passé, l'historiographie de la traduction, le présent et l'avenir.

\section{Références bibliographiques}

Anghelescu, Mircea. «Observaţii cu privire la traducerile româneşti din Halima în secolul al XVIII-lea». In: Limba Română. 1974, Vol. XXIII, nr. 1: 25-28. Anghelescu, Mircea. «Prima traducere românească din Halima». In: Limba Română. 1972, Vol. XXI, nr. 3: 262-266.

Barbu, Violeta. "Cele mai vechi traduceri din Voltaire». In: Limba română. 1987, Vol. (I), 36, nr. 6: 525-535.

*** Bibliografia relaţiilor literaturii române cu literaturile străine în periodice (1859-1918), vol. I-III, lucrare coordonată de Ioan Lupu şi Cornelia Ştefănescu, Editura Academiei R.S.R, 1980-1985.

*** Bibliografia relaţiilor literaturii române cu literaturile străine în periodice (1919-1944), vol. IV-VII, Institutul de Istorie şi Teorie Literară „George Călinescu”, autori: Ana - Maria Brezuleanu, Ileana Mihăilă, Viorica Niscov, Michaela Şchiopu, Cornelia Ştefănescu, prefaţă de Dan Grigorescu, Bucureşti, Editura Academiei Române,1997-2002.

*** Bibliografia traducerilor din literaturile slave (1945-2011) [Bibliographie des œuvres littéraires slaves traduites en roumain]. Geambaşu, Constantin (coord.). Bucureşti: Editura Universității din București, 2011.

Camariano, Ariadna. Spiritul revoluţionar francez şi Voltaire în limba greacă şi română [L'esprit révolutionnaire français et Voltaire dans les langues grecque et roumaine]. Bucureşti : Tiparul «Cartea românească», 1946.

Camariano-Cioran, Ariadna. Relaţii româno-elene: studii istorice şi filologice (secolele XIV - XIX) [Relations gréco-roumaines. Études d'histoire et de philologie (XIVe - XIXe siècles)]. Bucureşti : Editura Omnia, 2008.

Călinescu, George, Istoria literaturii române dela origini până în prezent [Histoire de la littérature roumaine, des origines à nos jours]. Bucureşti: Fundația pentru Literatură și Artă, 1941.

Cioculescu, Şerban. Istoria literaturii române. Epoca marilor clasici prezent [Histoire de la littérature roumaine. Époque de grands classiques]. Bucureşti: Editura Academiei, 1973.

Cornea, Paul. De la Alexandrescu la Eminescu. Aspecte-Figuri-Idei. Bucureşti : Editura pentru Literatură, 1966.

Cosco, Olga. «Primele cărţi franceze traduse în româneşte. Istoria lui Carol XII de Voltaire»[Les premiers livres français traduits en roumain. L'Histoire de Charles XII de Voltaire]. In: Cercetari literare. 1934, Vol. I: 102-114.

Delisle, Jean. "Réflexions sur l'historiographie de la traduction et ses exigences scientifiques ». In : Équivalences (ISTI, Bruxelles), vol 26, no 2 et vol. 27, no 1, 1997-1998: 21-43.

Delisle, Jean. «L'évaluation des traductions par l'historien». In : Meta: journal des traducteurs, Vol. 46, $\mathrm{n}^{\circ} 2$, juin 2001: 209-226. URL: 
http://id.erudit.org/iderudit/oo2514ar. Consulté le 3 juillet 2010.

D'hulst, Lieven. Essais d'histoire de la traduction. Avatars de Janus. Paris : Classiques Garnier, 2014.

Fecioru, D. Bibliografia traducerilor în româneste din literatura patristica.[Bibliographie des traductions roumaines de la littérature patristique]. Vol I .Fascicola I: Epoca dela 1691 pâna la 1833. Bucuresti: Institutul de Arte grafice Bucovina, 1937.

Gafton, Alexandru. De la traducere la norma literară. Contribuia traducerii textului biblic la constituirea vechii norme literare.[De la traduction à la norme littéraire]. Iași : Editura Universității «Al. I. Cuza» din Iași, 2012.

Gheţie, Ion et Mareş, Alexandru. Cele mai vechi cărţi populare în literatura română. Floarea darurilor şi Sindipa. Bucureşti : Editura Minerva, 1996.

Gheţie, Ion. Istoria limbii române literare. Bucureşti : Editura Ştiinţifică şi Enciclopedică, 1978.

Giurginca, Ioana-Simina. Le discours traductologique in statu nascendi dans les préfaces des traducteurs roumains de français-source au XIXème siècle. Timisoara: Universitatea de Vest din Timisoara, mémoire de dissertation, 2012.

Grimm, Petre. Scrieri de istorie literara [Écrits d'histoire littéraire]. Ediție îngrijită, prefaţă, tabel cronologic şi notă asupra ediţiei de Liana Muthu. ClujNapoca: Eikon \& Scriptor, [1916-1943]2012.

Heliade Rădulescu, Ion. Gramatica românească. Ediţie şi studiu de Valeria Guţu Romalo. Bucuresti : Editura Eminescu, [1828]1980.

Horguelin, Paul. L'Anthologie de la manière de traduire. Montréal : Linguatech, 1981.

Iorga, Nicolae. Istoria literaturii româneşti din veacul al XIX-lea - de la 1821 innainte [Histoire de la littérature roumaine du XIX ${ }^{\text {e }}$ siècle]. Bucureşti-Vălenii de munte : Minerva/Tip. Neamul românesc, 1907-1909.

Iorga, Nicolae. Histoire des relations anglo-roumaines. Iaşi: Imprimerie „Progresul”, 1917.

Iorga, Nicolae. Traducerile din limba franceză în literatura românească [Traductions roumaines du français]. Vălenii de munte: Tipografia Datina românească, 1936.

Isar, Nicolae. Opera și ideile lui Simeon Marcovici. Autorul „retoricii” şi al traducerilor din litera. In: N. Isar, Sub semnul romantismului. De la domnitorul Gheorghe Bibescu la scriitorul Simeon Marcovici. Bucuresti : Universitatea din Bucuresti, 2003.

Ivănescu, Gheorghe. Istoria limbii române. [Histoire de la langue roumaine] Iaşi : Editura «Junimea», 1980.

Ivaşcu, George. Istoria literaturii române prezent [Histoire de la littérature roumaine]. Bucureşti: Editura Ştiinţifică. 1969.

Jacquier, Henri. Babel mit viu [Babel, mythe vivant]. Ediţie îngrijită, prefaţă, antologie, note şi comentarii de M. Muthu. Cluj-Napoca: Editura Dacia, 1991.

Kogălniceanu, Mihail. Despre literatură [Sur la littérature]. Bucureşti : E.S.P.L.A., [1840-1844] 1956. Culegere, note şi cuvânt înainte de Dan Simonescu (p. 3-18). .

Ladmiral, Jean-René. Traduire. Théorèmes pour la traduction. Paris : Gallimard, [1979] 2010.

Lenz, Hélène. La Traduction et la modernité littéraire. In: [éd.] Centre d'Étude de l'Europe Médiane, Histoire de la traduction en Europe médiane. Paris : INALCO, 2011. URL: http://histrad.info/index.php?option=com_ 
content\&view $=$ article\&id $=365$ :la-traduction-et-la-modernite-litteraire\&catid = 54:roumain\&Itemid $=85$. [Consultation: le 0502 2013.]

Lenz, Hélène. La Traduction des textes religieux. In: [éd.] Centre d'Étude de l'Europe Médiane, Histoire de la traduction en Europe médiane. Paris : INALCO, 2011. http://histrad.info/index.php?option=com_content\&view=category\&layout=b $\log \&$ id $=54 \&$ Itemid $=85$. [Consultation: le 1803 2013.]

Lenz, Hélène. La Traduction et la formation de la littérature profane. In: [éd.] Centre d'Étude de l'Europe Médiane, Histoire de la traduction en Europe médiane. Paris: INALCO, 2011. URL: http://histrad.info/index.php?option= com_content\&view=article\&id=333:la-traduction-et-la-formation-de-la-

litterature-profane\&catid=54:rou. [Consultation : le 0105 2014.]

Loría-Rivel, Gustavo-Adolfo. Pentateuhul: probleme de traducere a textului biblic. [Le Penteteuque: problèmes de traduction]. Iași: Editura Universității « Al. I. Cuza » din Iași, 2004.

Lovinescu, Eugen. Istoria literaturii române contemporane [Histoire de la littérature roumaine contemporaine]. Vol I-V. București: Editura Ancora Benvenisti, 1926-1929.

Lungu-Badea, Georgiana. Idei și metaidei traductive românești (secolele al XVI-lea -al XXI-lea) [Idées et métaidées traductives roumaines]. Timişoara: Editura Eurostampa, 2013.

Lungu-Badea, Georgiana. Scurtă istorie a traducerii. Repere traductologice [Brève histoire de la traduction. Repères traductologiques]. Timişoara: Editura Universităţii de Vest, 2007.

Lungu-Badea, Georgiana. "Cine, ce şi cum traduce? Intenții. Subiecte. Metode» [Qui traduit? Quoi et comment traduit-on? Intentions, sujets; méthodes]. In: Romània între interculturalitate şi identitate: Spaţii romanice europene şi extraeuropene. [éd.] C. Timoc. Szeged: University Press of Szeged, 2015. Les Actes de la 3 édition de la CICCRE, des 3 et 4 octobre 2014.

Lungu-Badea, Georgiana. «Übersetzungsmethoden im Rumänischen im 18. und 19. Jahrhundert. Politische, sprachliche, ethische und ästhetische Problemstellungen». In: „Traducerile au de cuget să îmblînzească obiceiurile ..... Rumänische Übersetzungsgeschichte - Prozesse. Produkte. Akteure. [éd.] M. Jeanrenaud, J. Richter et L. Schippel. Berlin : Frank \& Timme, 2014: 3360.

Lungu-Badea, Georgiana. «Le rôle des langues intermédiaires dans la réception de la littérature française traduite en roumain au XVIII e siècle». Tribune internationale des langues vivantes. Dossier: «Recherches en traductologie: Les langues intermédiaires». 2011, Vol. 51: 42-51.

Lungu-Badea, Georgiana. Rolul dicţionarelor în desăvîrşirea limbii şi a traducerii [Le rôle des dictionnaires dans l'achèvement de la langue et de la traduction]. In: Un capitol de traductologie. Studii de istorie a traducerii (III). [ed.] G. Lungu-Badea. Timişoara: Editura Universităţii de Vest, 2008: 259270.

Lupu, Coman. Lexicografia românească în procesul de occidentalizare latinoromanică a limbii române moderne (1780-186o) [La lexicographie roumaine et le processus de latinisation et de romanisation du roumain moderne (17801860)]. Bucureşti: Editura Logos, 1999.

Macedonski, Alexandru. Opere II. Dramaturgie. Traduceri și adaptări în limba română. Versuri, proză, dramaturgie în limba franceză. [Oeuvres II. Dramaturgie. Traductions et adaptaions en roumain. Vers, prose, dramaturgie 
en français]. Édition soignée par M. Colojoară, préface d’Eugen Simion. București: Editura Fundației Naționale pentru Ştiință şi Artă, 2004

Mateescu, C.N. «Însemnări despre profesorul craiovean Stanciu Căpăţîneanu». In: Analele Olteniei. 1925, Vol. IV: 21-22.

Moraru, Alexandra. Floarea Darurilor: Text stabilit, studiu filologic. In: I. Gheţie și Al. Mareş. Cele mai vechi cărţi populare în literatura română. Floarea darurilor şi Sindipa. Bucureşti : Editura Minerva, 1996: 15-74.

Munteanu, Eugen. Studii de lexicologie biblică [Études de lexicologie biblique]. Iaşi : Editura Universităţii «Al. I. Cuza», Iaşi, 1995.

Munteanu, Ştefan et Ţâra, Vasile. Istoria limbii române literare [L'histoire de la langue roumaine littéraire]. Bucureşti : Editura Didactică şi Pedagogică, [1978] 1983.

Nicolescu, Aurel. «Observaţii asupra neologismelor din „Însemnare a călătoriei mele" a lui Dinicu Golescu»[Observations sur les néologismes chez D. Golescu]. In: *** Contribuţii la istoria limbii române literare în secolul al XIX-lea,. Bucureşti : Editura Academiei R.S.R., 1958, Vol. II: 5-53.

Nistor, I. «O traducere din Voltaire în arhiva mănăstirii Putna » [Une traduction de Voltaire aux archives du monastère de Putna]. In: Junimea literară. 1939, Vol. XXVIII: 1-12.

Obrocea, Nadia. Influenţa traducerilor româneşti din limba franceză în secolele al XVIII-lea şi al XIX-lea. In: [ed.] Georgiana Lungu-Badea. Un capitol de traductologie românească. Studii de istorie a traducerï (III). Timişoara : Editura Universităţii de Vest, 2008: 221-246.

Piru, Al. Istoria literaturii române de la origini până la 1830. Bucureşti : Editura Ştiinţifică şi Enciclopedică, 1977.

Philippide, Alexandru. Introducere în istoria limbei şi literaturei romîne [Introduction à l'histoire dela langue et la littérature roumaine], Editura librariei Frații Saraga, 1888.

Puşcariu, Sextil, Istoria literaturii române I. Epoca veche [Histoire de la littérature roumaine. L'époque ancienne]. Bucureşti: Editura Eminescu, 1989 [Sibiu, Editura Asociaţiunii, 1920].

Reischel, Arthur. "Bibliografia traducerilor din literatura maghiara în limba româna » [Bibliographie des traductions roumaines de la littérature hongroise]. In: Sesiune ştiinţifică de bibliologie şi documentare: Comunicări şi discuţii. Volume 1. Bucuresti: Editura Academiei R.P.R., 1957: 67-68.

*** [RTRI] Repertoriul traducătorilor români de limbă franceză, italiană, spaniolă (secolele al XVIII-lea şi al XIX-lea). Studii de istorie a traducerii (I) [Répertoire des traducteurs roumains de langue française, italienne, espagnole (XVIIIe et XIXe siècles)]. Lungu-Badea, G. (coord.). Timişoara : Editura Universităţii de Vest, 2006..

*** [RTRII] Repertoriul traducerilor româneşti din limbile franceză, italiană, spaniolă (secolele al XVIII-lea şi al XIX-lea). Studii de istorie a traducerii (II) [Répertoire des traductions roumaines des langues française, italienne, espagnole (XVIII e et XIX ${ }^{\mathrm{e}}$ siècles)]. Lungu-Badea, G. (coord.). Timişoara : Editura Universităţii de Vest, 2006.

Tenchea, Maria et Lungu-Badea, Georgiana. «Perspectives traductologiques roumaines ». In : [éd.] Michel Ballard. Qu'est-ce que la traductologie? Arras : Presses de l'Université d'Artois, 2007.

*** Un capitol de traductologie românească. Studii de istorie a traducerii (III)[Un chapitre de traductologie roumaine (XIX ${ }^{\mathrm{e}}$ siècle)]. Lungu-Badea, G. (coord.). Timişoara : Editura Universităţii de Vest, 2008. 
Ursu, N. A. Formarea termenilor ştiinţifici româneşti. Bucureşti : Editura Ştinţifică, 1962.

Ursu, N. A. «Şcoala de traducători români din obştea stareţului Paisie de la mănăstirile Dragomirna, Secu şi Neamţ» [L'École de traduction roumaine de Paisie, aux monastères de Dragomirna, Secu et Neamţ]. In: Teologie şi viaţa, 1994, Vol. VI (LXX), nr. 11-12: 58-83.

Ursu, N. A. «Traducerile logofătului Toma Dimitriu de la Mitropolia din Iaşi»[Les Traductions du chancelier Toma Dimitriu de l'Église Métropolitaine de Iaşi]. Almanahul Institutului de Istorie «A. D. Xenopol». 1999, Vol. XXXVI: 7-24. 